\title{
Early prediction system using neural network in Kelantan River, Malaysia
}

\begin{abstract}
Flood is a major disaster that happens around the world. It has caused the loss of many precious lives and destruction of large amounts of property. The possibility of flood can be determined depends on many factors that consist of rainfall, water flow rate and water level. This project aims to design a water level prediction system which is used to analyze the Kelantan River water level based on Sokor River, Galas River and Lebir River flow rate and rainfall of at Ldg. Kuala $\mathrm{Nal}$ and Ldg. Kenneth. The system utilizes neural networks in predicting the water level for 5 hours ahead. This system has 5 inputs and 1 output prediction. This prediction system focusses on comparing the conventional method and the Neural Network Autoregressive with Exogenous Input (NNARX) system in determining the possibility of flood. The result shows that the NNARX can predict the water level of Kelantan River much more better compared to conventional method. The performance of the system is based on the value of the means square error (MSE). The MSE of the conventional method is 0.2550 meanwhile for NNARX is $1.342 \times 10 \overline{1} 4$.
\end{abstract}

Keyword: Neural network; Neural network autoregressive with exogenous input (NNARX); Flood prediction model 